\title{
Acknowledgment to Reviewers of Diseases in 2020
}

\section{Diseases Editorial Office}

MDPI AG, St. Alban-Anlage 66, 4052 Basel, Switzerland

Peer review is the driving force of journal development, and reviewers are gatekeepers who ensure that Diseases maintains its standards for the high quality of its published papers. Thanks to the cooperation of our reviewers, in 2020, the median time to first decision was 17.6 days and the median time to publication was 35.5 days. The editors would like to express their sincere gratitude to the following reviewers for their precious time and dedication, regardless of whether the papers were finally published:

Adey, Deborah

Ai, Yong

Akil, Luma

Annunziata, Giuseppe

Asakawa, Mitsuhiko

Atehortua, Nelson A. De La Pena

Ayala-Zavala, J. F.

Berndt, Michael C.

Bhuiyan, Azad

Bondy, Stephen C.

Bresciani, Elena

Brieño-Enriquez, Miguel

Brown, Mark

Buchet, Rene

Byeon, Haewon

Cappello, Francesco

Careem, Faizal

Carlini, Célia R.

Casella, Giovanni

Castiglia, Paolo

Chatzikyrkou, Christos

Chen, Guoxun

Chen, Tai-Heng

Cheng, Hung-Chi

Chowdhury, Imran Hussain

Chreptowicz, Karolina

Conn, David Bruce

Conti, Michele

Cretoiu, Dragos

Cunningham, Lisa

Dampier, Carlton

Dario, Brunetti

De Spiegelaere, Ward

De Villiers, Marianne

Dean, Frank

Deb, Subrata

Huang, Kai
Dec, Marta

Dey, Priyankar

Di Micco, Pierpaolo

Efird, Jimmy T.

Erdmann, Kati

Escolà-Gil, Joan Carles

Faita, Francesco

Fiorucci, Stefano

Fonseca, Kevin

Franzoni, Ferdinando

Fujita, Toshitsugu

Furmańczyk, Konrad

Galassi, Andrea

Gao, Bei

Garinis, George

Gazzonis, Alessia Libera

Giacobini, MaiBritt

Giesbrecht, Gerald F.

Gobba, Fabrizio Maria

Gordon, Catherine A.

Guerini, Franca R.

Guerriero, Giulia

Guetl, Katharina

Gutenbrunner, Christoph

Hamaguchi, Masahide

Hanyaloglu, Aylin C.

Hayashida, Kyoko

He, Jin-Sheng

Heleniak, Zbigniew

Hiebert, Paul

Hinds, Rupert

Holt, Stephen

$\mathrm{Hsu}$, Lewis

$\mathrm{Hu}$, Andy

$\mathrm{Hu}$, Xiaoyu

Huang, Helen J.

Pace, Fabio 
Ismail, Hanafy Mahmoud

Junichi, Kitanaka

Kadotani, Hiroshi

Kandeel, Mahmoud

Kane, Eleanor

Kashfi, Khosrow

Kato, Takamitsu A

Keenan, Jeffrey A.

Kenmochi, Takashi

Kim, $\mathrm{Ka}$

Kim, Won Young

Klinger, Marian

Kołodziejczak, Michalina

Konhilas, John

Kouji, Harada

Krintus, Magdalena

Kuryłowicz, Alina

Lamichhane, Santosh

Lau, Wei Ling

Lazarevic-Pasti, Tamara

Li, Binxing

Li, Tieshi

Lichtenauer, Michael

Lin, Cheng-Wen

Lin, Pei-Hui

Liu, Shichao

Lu, Jun

Maddelein, Marie-Lise

Marcoulides, George A.

Martín Casanueva, Miguel Angel

Mascellino, Maria Teresa

Mastrangelo, Stefano

Maugeri, Andrea Giuseppe

Meerson, Ari

Mesch, Gustavo

Metzinger, Laurent

Mizuno, Masashi

Mlocicki, Daniel

Modesti, Alessandra

Moles, Laura

Moret-Tatay, Carmen

Muniyan, Sakthivel

Narayan, Prema

Nastasi, Claudia

O'Hara, Steven

Obermüller, Nicholas

Okech, Bernard

Osuna, Eduardo

Owino, Simon Odera

Venusia, Covelli
Pacenti, Monia

Palomba, Letizia

Palomino-Moral, Pedro Ángel

Pande Katare, Deepshikha

Pasaje, Charisse Flerida A.

Pasquinelli, Gianandrea

Patel, Deven

Pence, Brandt

Pérez-García, Selene

Phalak, Poonam

Piana, Andrea

Pleass, Henry

Prado-Cabrero, Alfonso

Rathinasabapathy, Anandharajan

Ravaioli, Stefano

Reynolds, Mary

Ribaldone, Davide

Romine, William

Rosado, Carlos

Rosenthal, Ken

Roubertoux, Pierre

Scapellato, Maria Luisa

Schermer, Julie Aitken

Sergi, Pier Nicola

Sergunin, Alexander

Sharma, Isha

Sinigaglia, Alessandro

Snelling, William

Sorahan, Tom

Speeckaert, Marijn

Splichal, Igor

Sridhar, Subbaramiah

Stack, Gary

Stieger, Bruno

Sun, Chongkui

Suzuki, Tohru

Tajiri, Yuji

Tamayo-Ortiz, Marcela

Tavoschi, Lara

Teschke, Rolf

Thomas, Roger

Timson, Benjamin F.

Toietta, Gabriele

Tomsone, Signe

Tonetti, Lorenzo

Tripathi, Manish

Tünde, Tarr

Usai Satta, Paolo

Vaandrager, Arie B.

Wang, Xiangru 
Veraldi, Stefano

Vezzoli, Valeria

Vigilato, Marco Antonio Natal

Vukojevic, Katarina

Wang, Chih-Hao

Wang, Cuicui
Wei, Chengguo

$\mathrm{Xu}$, Wen Tao

Yang, Jiann-Jou

Yeligar, Samantha M.

Zhang, Chengxian

Zhang, Duo 